\title{
Health professionals' ability to recognise body weight status of adults and children: the outcomes of the Weight Care Project, an all-Ireland study
}

\author{
S. A. Moorhead ${ }^{1}$, A. Gallagher ${ }^{2}$, D. Hazlett ${ }^{1}$, V. Coates $^{3}$, K. Murphy ${ }^{4}$ and G. Nolan ${ }^{5}$ \\ ${ }^{1}$ School of Communication, University of Ulster, BT37 OQB, UK, ${ }^{2}$ School of Biomedical Sciences, University of Ulster, \\ BT52 1SA, UK, ${ }^{3}$ School of Nursing, University of Ulster, BT52 1SA, UK, ${ }^{4}$ School of Nursing and Midwifery, \\ National University of Ireland, Republic of Ireland and ${ }^{5}$ Department of Health Promotion, National University of Ireland, \\ Republic of Ireland
}

Obesity is a key public health issue on the island of Ireland $(\mathrm{IoI})^{(1,2)}$. Health professionals could have a vital role in initiating recognition of elevated body weight status amongst the population and increasing the public's awareness of overweight/obesity. However, the accuracy with which health professionals assess body weight status is not known. One of the objectives of the Weight Care Project was to assess health professional groups' ability to identify body weight categories within adults and children on an all-Ireland basis.

An online assessment tool was developed for the purposes of this study to assess 128 (power calculation) health professionals' ability to identify the body weight category of adults and children (20 model case studies of different ages [children, teenagers, young adults, middle-aged adults, older adults] and weight categories [underweight, normal weight, overweight, obese]). Each model was presented three times: Screen $1-$ video of the model rotating $360^{\circ}$ in their own clothes with information on their gender, age and height; Screen 2 as per Screen 1 but now in the project's clothes (shorts and t-shirt); Screen 3 - as per Screen 2 but with additional information on weight. Case studies were presented in random order. Following the presentation of each screen, health professionals were asked to identity the body weight category of the model. The target health professional groups were as follows: 1. Public Health Nurses (Community; postnatal home and clinic visits and developmental checks); 2. Public Health Nurses (Schools); 3. GPs and Practice Nurses (Primary Care); 4. Occupational Health Nurses (Workplace). Ethical approval was obtained from seven ethical/research governance committees throughout the $\mathrm{IoI}^{(3)}$.

From the 128 health professionals who completed this study, the breakdown of health professional groups in the sample reflected the ratio of these health professionals on the IoI. Health professionals who accurately recognised normal weight status in individuals wearing their own clothes across the different age categories of the models ranged from 56\%-95\%. However, the ability to accurately recognise underweight, overweight, and obese body weight status categories was poorer. As health professionals who accurately recognised obese models wearing their own clothes across the different age categories of the models ranged from $2 \%-50 \%$. Overall, health professionals were significantly $(P<0.001)$ more accurate at assessing body weight category of individuals when presented in standardised project clothes (rather than the model's own clothing) and when provided with information on body weight. Overall, health professionals with lower BMI $(P<0.05)$ and increasing age $(P<0.05)$ were significantly more accurate in assessing body weight categories. Health professionals reported a lack of confidence in assessing bodyweight status, and were significantly $(P<0.001)$ less confident in assessing body weight in children compared to adults. Health professionals reported limited training to-date, and the need for training in assessing body weight status. Some significant $(P<0.05)$ differences were observed between the different health professional groups.

In conclusion, a large proportion of health professionals failed to correctly classify body weight categories of a range of study models. Failure to recognise overweight and obesity presents a major barrier to obesity prevention and management on the IoI. These findings support further focus on training in assessing body weight status as part of obesity prevention strategies amongst health professionals on the IoI.

This research was funded by safefood, the Food Safety promotion Board, under Grant No. 08/2009.

1. Irish Universities Nutrition Alliance (2012) The National Adult Nutrition Survey. Available at: http://www.iuna.net/ Accessed 28 February 2012.

2. Department of Health, Social Services and Public Safety (2011) Health Survey Northern Ireland: First Results from the 2010/2011 Survey. Belfast: Department of Health, Social Services, and Public Safety.

3. Moorhead SA, Coates V, Hazlett D et al. (2011) BMC Public Health 11, 202. 